\title{
A longitudinal analysis of pulmonary function in rats during a 12 month cigarette smoke exposure
}

\author{
J.L. Wright*, J-P. Sun*, S. Vedal**
}

A longitudinal analysis of pulmonary function in rats during a 12 month cigarette smoke exposure. J.L. Wright, J-P. Sun, S. Vedal. (CERS Journals Ltd 1997.

ABSTRACT: We wanted to examine the longitudinal effects of chronic cigarette smoke exposure, and to determine whether the chronic alterations in pulmonary function induced by long-term cigarette smoke exposure in an animal model could be predicted by initial or early alterations in function.

A group of Sprague Dawley rats was exposed to the smoke of 7 cigarettes $^{-1 a y}{ }^{-1}$ for 5 days week $^{-1}$ during a total period of 12 months. Lung volume, flow-volume curves and pressure-volume curves were recorded at baseline, and after 2, 4, 8 and 12 months of smoke exposure. A control group of rats was subjected to the same regimen of testing, but was not exposed to smoke.

Thirteen rats completed the study in the smoke-exposed group and seven rats in the control group. We found that chronic exposure to cigarette smoke produced early abnormalities in pulmonary function, with the forced expiratory volume in one second/forced vital capacity (FEV1/FVC) ratio showing an acceleration of ageing effect, particularly between 4 and 8 months of exposure. In this model, although the two groups had significantly different airflow after 12 months, the initial values in each group were remarkably similar, and we could not identify any pulmonary function test which had predictive value.

We conclude that longitudinal studies of cigarette smoke exposure in this rat model allow better characterization of the nature and time course of the effects of smoking on the lung.

Eur Respir J 1997; 10: 1115-1119.

Depts of *Pathology and **Medicine, University of British Columbia, Vancouver, Canada.

Correspondence: J.L. Wright

Dept of Pathology

2211 Wesbrook Mall

Vancouver

B.C. V6T 2B5

Canada

Keywords: Cigarette smoke predictive value

pulmonary function tests

Received: July 161996

Accepted after revision January 141997

Supported by grants from the B.C. Health Research Foundation and the B.C. Lung Association.
In our laboratory, we have previously developed a guinea-pig model of cigarette smoke-induced alterations in pulmonary function [1, 2]. Although this model has proved successful in that we have been able to show clear evidence of airflow obstruction and emphysematous lung destruction in smoke-exposed animals, it has also illustrated the problems inherent in such kinds of cross-sectional studies. In the guinea-pig, there was significant interanimal variation in pulmonary function, even when accounting for animal age and weight [3]. Furthermore, we found that our cross-sectional studies were biased by a "healthy smoker" effect, whereby the animals that were most affected by cigarette smoke tended to die before the end of the experiment; the final results were, thus, derived from the animals with the least disease [2, 4]. Finally, the use of a single pulmonary function end-point does not allow for analysis of the temporal profile of the development of abnormalities, or for analysis of results that might predict response to cigarette smoke.

It was for these reasons that, in the present study, a longitudinal study design was chosen, whereby each animal was used as its own control and function tests were performed at multiple time-points, thus making it possible to track each animal's function by calculating a percentage change from control values. This type of longitudinal analysis also had the advantage that it was possible to ascertain whether any baseline or early function test could be predictive of ultimate function. The primary objective of this study was to characterize the long-term effect of cigarette smoke exposure on a large array of pulmonary function tests that reflect different pathophysiological derangements in the lung. A secondary objective was to ascertain whether any pulmonary function test could be predictive of final pulmonary function.

Although other workers have reported success in intubation of guinea-pigs [5, 6], we found this technique hampered by pronounced laryngospasm, resulting in an unacceptable death rate. We therefore performed our study in the Sprague Dawley rat, an animal which is easily intubated. Although we had previously shown that there was little variation in pulmonary function tests repeated at the same time [1], we had not been able to test reproducibility over a period of several days. An initial objective of the present study was, therefore, to show this reproducibility, thereby allowing for the longitudinal analysis of pulmonary function.

\section{Methods}

To ascertain whether the pulmonary function tests used in this model were reproducible, 10 Sprague Dawley 
rats weighing $400-510 \mathrm{~g}$ (mean \pm SD $447 \pm 30 \mathrm{~g}$ ) were utilized. In this portion of the study, three sets of pulmonary function tests were performed: baseline, Day two, and Day three.

In the 12 month study, Sprague Dawley rats that initially weighed $317 \pm 18 \mathrm{~g}$ were used. Baseline function tests were performed, and the animals were then randomly assigned to either control (non-smoke-exposed) or smoke-exposed groups. Pulmonary function was analysed at baseline, 2, 4, 8 and 12 months.

\section{Pulmonary function tests}

The animals were anaesthetized using a combination of intraperitoneal diazepam $\left(5 \mathrm{mg} \cdot \mathrm{kg}^{-1}\right)$ and intramuscular Innovar Vet $\left(0.4 \mathrm{~mL} \cdot \mathrm{kg}^{-1}\right)$, and intubated with a 14 gauge intravenous cannula [4], using a paediatric laryngoscope and a tilted table. After intubation, the rats were placed in a pressure sensitive $7.5 \mathrm{~L}$ small animal plethysmograph, and ventilated at 80 breaths $\cdot \mathrm{min}^{-1}$ with a tidal volume of approximately $1.5 \mathrm{~mL}$. A water-filled oesophageal tube (PE 240) with a multiholed tip was used to measure pleural pressure; transpulmonary pressure was calculated as the difference between mouth and pleural pressure.

Pulmonary function tests were performed according to our usual protocol [1]. After measurement of functional residual capacity (FRC), the rats were given supplementary doses of Innovar Vet, and were rendered apnoeic by hyperventilation. Pressure-volume curves were constructed by deflation to $-30 \mathrm{cmH}_{2} \mathrm{O}$ (expiratory reserve volume (ERV)), inflation to $+30 \mathrm{cmH}_{2} \mathrm{O}$, and deflation to $-30 \mathrm{cmH}_{2} \mathrm{O}$ (vital capacity (VC)). Two inflation-deflation procedures were performed prior to measurement of the curve, and calculation of lung volumes. Static lung compliance $(C \mathrm{~L}, \mathrm{st})$ was calculated between $\mathrm{FRC}$ and $10 \mathrm{cmH}_{2} \mathrm{O}$ transpulmonary pressure. A flow-volume curve was constructed by inflation to $+30 \mathrm{cmH}_{2} \mathrm{O}$, and rapid deflation to $-50 \mathrm{cmH}_{2} \mathrm{O}$ pressure. From this curve, forced vital capacity (FVC), forced mid-expiratory flow (FEF25-75), forced expiratory volume in one second (FEV1), and peak expiratory flow (PEF) were calculated. The flow volume curve itself was plotted, using the volumes at $95-30 \%$ total lung capacity (TLC) as the independent variable.

Following the pulmonary function tests, the animals were kept warm, allowed to recover from the anaesthetic, and were extubated. They were housed in a laminar hood in standard rat cages with sterile paper pellets as bedding, and were allowed free access to rat chow and water.

\section{Cigarette smoke exposure}

We utilized our standard smoke exposure system [1]. In this system, exposure to the smoke of 7 cigarettes.day ${ }^{-1}$ causes a chronic carboxyhaemoglobin level of appro-ximately 4\%, a value similar to that found in human chronic cigarette smokers [7], and has been shown to produce emphysema in guinea-pigs [1]. In brief, the system consists of a vented nose only chamber, into which serial aliquots of $20 \mathrm{~mL}$ of fresh cigarette smoke were injected, with each cigarette consumed over a period of approximately $10 \mathrm{~min}$. The animals received the smoke of 7 cigarettes $\cdot$ day $^{-1}$ for 5 days $\cdot$ week $^{-1}$ during a 12 month total time period.

\section{Statistical analysis}

The data were analysed using the SYSTAT [8] and Statistical Analysis System (SAS) [9] statistical packages. A multiple analysis of variance (MANOVA) was used both to analyse the reproducibility of the pulmonary function measurements over 3 days, and to test for the effect of cigarette smoke exposure on the repeated measurements over 12 months. For reproducibility, Hotelling's $\mathrm{T}^{2}$ statistic was used to determine whether significant differences in the three lung function measurements (Day 0,2 and 3 ) were present. The Hotelling $\mathrm{T}^{2}$ test is the analogue of the paired t-test for the case where more than two measurements are made on each experimental animal, and accounts for the correlation between tests within each animal.

For testing the smoke effect, the five pulmonary function measurements (Month 0, 2, 4, 8 and 12) were modelled as a function of time and group (smoke-exposed and control). An effect of cigarette smoking was deemed to be present when a statistically significant interaction between time and group was present; i.e. there was an effect of smoking when the effects of time differed between the smoke-exposed and control groups. A "profile" contrast analysis [10] was performed to identify for which of the four adjacent month pairs $(0-2$, 2-4, 4-8, and 8-12 months) significant effects for time and smoking were present. Similar analyses were performed after dividing each pulmonary function measurement by the animal's weight at the time of the measurement. A Bonferroni correction was applied to account for the four comparisons made in the profile analysis. A p-value of less than 0.05 was considered statistically significant.

\section{Results}

\section{Reproducibility study}

Table 1 presents the mean \pm SD values for the lung volumes, which were derived from the pressure-volume curves (data not shown), whilst table 2 presents the mean \pm SD values for the flow data, which were calculated from the flow-volume curve. Each table also indicates the pvalue corresponding to the Hotelling's $\mathrm{T}^{2}$ statistic. No

Table 1. - Reproducibility study: lung volume analysis

\begin{tabular}{lcccc}
\hline Test & Day 1 & Day 2 & Day 3 & p-value \\
\hline VC mL & $16.7 \pm 2.1$ & $16.8 \pm 2.1$ & $17.2 \pm 2.1$ & 0.29 \\
FRC mL & $6.7 \pm 0.3$ & $6.7 \pm 0.2$ & $6.8 \pm 0.3$ & 0.40 \\
RV mL & $5.2 \pm 0.7$ & $4.5 \pm 0.5$ & $4.7 \pm 0.8$ & 0.07 \\
CL,st mL.cm & $0.92 \pm 0.18$ & $0.88 \pm 0.14$ & $0.95 \pm 0.17$ & 0.23 \\
\hline
\end{tabular}

Values are presented as mean \pm SD. VC: vital capacity; FRC: functional residual capacity; RV: residual volume; $C \mathrm{~L}$,st: static lung compliance. \#: from repeated measures analysis. 
Table 2. - Reproducibility study: flow rate analysis

\begin{tabular}{lcccc}
\hline Test & Day 1 & Day 2 & Day 3 & p-value \\
\hline PEF $\mathrm{mL} \cdot \mathrm{s}^{-1}$ & $149 \pm 12$ & $147 \pm 17$ & $147 \pm 14$ & 0.80 \\
FEF25-75 mL $\cdot \mathrm{s}^{-1}$ & $122 \pm 15$ & $121 \pm 21$ & $122 \pm 18$ & 0.91 \\
FEV1 mL & $5.6 \pm 1.3$ & $5.9 \pm 1.0$ & $5.7 \pm 1.0$ & 0.77 \\
FEV1/FVC & $30 \pm 6.6$ & $33 \pm 5.3$ & $31 \pm 4.4$ & 0.56 \\
\hline
\end{tabular}

Values are presented as mean \pm SD. PEF: peak expiratory flow; FEF25-75: forced mid-expiratory flow; FEV1: forced expiratory volume in one second; FVC: forced vital capacity. \#: from repeated measures analysis.

significant intra-animal differences were found for any of the parameters. The only test in which a near significant difference $(\mathrm{p}=0.07)$ was found was the residual volume (RV), which tended to decrease slightly in Days 2 and 3 . The flow data were stable over the 3 days, with p-values ranging from 0.56 (FEV1/FVC) to 0.77 (FEV1).

\section{Twelve month longitudinal analysis}

At the termination of the study, there were seven rats remaining in the control group and 13 remaining in the smoke-exposed group. Table 3 presents the data, expressed as percentage baseline value, for the control and smoke-exposed groups at each of the pulmonary function test times. Based on the raw values for each test, the MANOVA showed an overall effect of time for each of the pulmonary function tests $(p<0.001)$, and these effects remained when the analysis was repeated using weight as a corrective factor (values divided by weight). There was a significant overall smoke exposure effect for FRC, FEV1, and FEV1/FVC, as well as for weight (all $\mathrm{p}<0.001$ ), using the raw function values. After weight correction, an overall smoke exposure effect was also found for vital capacity (VC), RV, TLC, CL,st, PEF and FVC (all $\mathrm{p}<0.001$ ), but statistical significance for effects on FEV1 and FEV1/FVC were lost.

Significant interactions between time and smoke were found for $\mathrm{VC}(\mathrm{p}<0.02)$, FRC $(\mathrm{p}<0.001), \mathrm{RV}(\mathrm{p}<0.02)$,
TLC $(\mathrm{p}<0.001)$, weight $(\mathrm{p}<0.001)$, FEV1, $(\mathrm{p}<0.05)$ and FEV1/FVC $(p<0.05)$, when the raw data values were examined. After weight correction, statistically significant interactions persisted for VC $(\mathrm{p}<0.001)$, FRC $(p<0.001), R V(p<0.05)$, TLC $(p<0.001)$, and further interactions were identified for $C \mathrm{~L}$,st $(\mathrm{p}<0.006), \mathrm{PEF}$ $(\mathrm{p}<0.002)$, FEF25-75 $(\mathrm{p}<0.003)$ and FVC $(\mathrm{p}<0.001)$, while statistical significance for FEV1 and FEV1/FVC was lost.

The profile analysis was performed to identify the time periods between which effects occurred. Using the raw values, an overall time effect on lung volumes was found between baseline and 2 months for VC, RV, TLC, $C \mathrm{~L}, \mathrm{st}(\mathrm{p}<0.001)$ and FRC $(\mathrm{p}<0.01)$; after weight correction, effects remained for FRC $(\mathrm{p}<0.01)$, TLC $(\mathrm{p}<0.05)$ and RV $(\mathrm{p}<0.001)$. Time effect on flow values between baseline and 2 months was found on the raw values for PEF, FEF25-75, FVC, FEV1/FVC ( $<<0.001)$; after weight correction, effects remained $(p<0.001)$ for all but FVC, and became apparent for FEV1 $(p<0.001)$.

A time effect was found between 2 and 4 months on the raw values for VC, TLC, $C \mathrm{~L}$,st, FRC and FVC ( $\mathrm{p}<0.001)$; after weight correction, a time effect remained for FVC $(\mathrm{p}<0.05)$ only, and was joined by a significant effect for PEF $(\mathrm{p}<0.01)$.

A time effect between 4 and 8 months was found for VC, TLC, $C$ L,st and FRC $(\mathrm{p}<0.001), \mathrm{RV}(\mathrm{p}<0.01)$ and FVC $(\mathrm{p}<0.001)$, and FEV1/FVC $(\mathrm{p}<0.02)$; after weight correction, the time effect on FVC $(\mathrm{p}<0.05)$ and FEV1/ FVC $(\mathrm{p}<0.001)$ remained $(\mathrm{p}<0.01)$ and was joined by a significant effect on FEV1 $(\mathrm{p}<0.01)$.

A time effect between 8 and 12 months was found for FRC $(p<0.001)$, which was removed after weight correction, but replaced by an effect on $\mathrm{VC}(\mathrm{p}<0.05)$. Time effects between 8 and 12 months were also found for $\mathrm{FEV} 1$, and FEV1/FVC $(\mathrm{p}<0.001)$ and PEF $(\mathrm{p}<0.05)$ raw data, and these effects remained after weight correction and were joined by a significant effect on FEF25-75 $(\mathrm{p}<0.001)$. A significant time effect was found for weight between all adjacent time pairs $(p<0.001)$.

As shown in table 3, differences in lung volumes and $C \mathrm{~L}$,st between the control and smoke-exposed animals (evidence of cigarette smoke effect) were found in the

Table 3. - Pulmonary function (as \% baseline) over 12 months in control and smoke-exposed rats

\begin{tabular}{|c|c|c|c|c|c|c|c|c|c|c|c|c|}
\hline & \multicolumn{3}{|c|}{2 months } & \multicolumn{3}{|c|}{4 months } & \multicolumn{3}{|c|}{8 months } & \multicolumn{3}{|c|}{12 months } \\
\hline & C & S-E & $\begin{array}{l}\mathrm{p} \text {-value } \\
0-2 \text { mo }\end{array}$ & $\mathrm{C}$ & S-E & $\begin{array}{l}\text { p-value } \\
2-4 \text { mo }\end{array}$ & $\mathrm{C}$ & S-E & $\begin{array}{l}\text { p-value } \\
4-8 \mathrm{mo}\end{array}$ & C & S-E & $\begin{array}{r}\text { p-value } \\
8-12 \text { mo }\end{array}$ \\
\hline $\mathrm{VC}$ & $141 \pm 10$ & $162 \pm 12$ & $* * / * * *$ & $164 \pm 8$ & $183 \pm 13$ & NS/NS & $178 \pm 11$ & $194 \pm 17$ & NS/NS & $175 \pm 10$ & $197 \pm 15$ & NS/NS \\
\hline FRC & $121 \pm 7$ & $145 \pm 11$ & $* * * / * * *$ & $141 \pm 4$ & $151 \pm 8$ & $* * / \mathrm{NS}$ & $170 \pm 11$ & $167 \pm 12$ & $* * / *$ & $176 \pm 7$ & $176 \pm 12$ & NS/NS \\
\hline RV & $121 \pm 20$ & $133 \pm 24$ & NS/NS & $144 \pm 36$ & $124 \pm 26$ & NS/NS & $180 \pm 51$ & $132 \pm 28$ & NS/NS & $177 \pm 36$ & $155 \pm 22$ & $\mathrm{NS} / *$ \\
\hline TLC & $135 \pm 6$ & $154 \pm 12$ & $* * * / * * *$ & $157 \pm 6$ & $166 \pm 13$ & NS/NS & $177 \pm 9$ & $177 \pm 16$ & $* / *$ & $175 \pm 4$ & $184 \pm 14$ & $* / \mathrm{NS}$ \\
\hline$C \mathrm{~L}, \mathrm{st}$ & $135 \pm 28$ & $199 \pm 39$ & $* * / * * *$ & $204 \pm 24$ & $222 \pm 34$ & $* / \mathrm{NS}$ & $233 \pm 31$ & $253 \pm 42$ & NS/NS & $224 \pm 28$ & $265 \pm 43$ & NS/NS \\
\hline Weight & $168 \pm 13$ & $142 \pm 9$ & $* * *$ & $195 \pm 18$ & $160 \pm 14$ & NS & $216 \pm 22$ & $179 \pm 14$ & NS & $229 \pm 26$ & $187 \pm 14$ & NS \\
\hline PEF & $123 \pm 8$ & $131 \pm 9$ & $\mathrm{NS} / * * *$ & $128 \pm 5$ & $132 \pm 13$ & NS/NS & $133 \pm 10$ & $137 \pm 9$ & NS/NS & $129 \pm 7$ & $130 \pm 10$ & NS/NS \\
\hline FEV1 & $101 \pm 33$ & $81 \pm 29$ & NS/NS & $114 \pm 32$ & $95 \pm 24$ & NS/NS & $116 \pm 38$ & $64 \pm 22$ & NS/NS & $66 \pm 24$ & $50 \pm 16$ & $* * / * *$ \\
\hline FEF25-75 & $121 \pm 10$ & $127 \pm 12$ & NS/** & $126 \pm 11$ & $133 \pm 17$ & NS/NS & $132 \pm 16$ & $139 \pm 11$ & NS/NS & $129 \pm 17$ & $137 \pm 13$ & NS/NS \\
\hline FVC & $140 \pm 9$ & $157 \pm 9$ & $* * / * * *$ & $154 \pm 11$ & $168 \pm 11$ & NS/NS & $162 \pm 10$ & $182 \pm 13$ & NS/NS & $170 \pm 10$ & $185 \pm 7$ & NS/NS \\
\hline $\mathrm{FEV} 1 / \mathrm{FVC}$ & $73 \pm 24$ & $52 \pm 19$ & NS/NS & $75 \pm 22$ & $57 \pm 16$ & NS/NS & $72 \pm 25$ & $35 \pm 13$ & NS/NS & $39 \pm 15$ & $27 \pm 9$ & $* * * * *$ \\
\hline
\end{tabular}

Values are presented as percentage of baseline value \pm SD. C: control group; S-E: smoke-exposed group; mo: months; TLC: total lung capacity; Ns: nonsignificant. For further definitions see legends to table 1 and 2. Statistical significance: profile analysis (analysis of the differences between $\mathrm{C}$ and S-E groups in the pulmonary function changes between months). The first symbol represents analysis using raw values; the second symbol represents analysis using weight correction. $*$ : $\mathrm{p}<0.05 ; * *$ : $\mathrm{p}<0.01 ; * * *$ : $\mathrm{p}<0.001$. 
baseline to 2 month time period, and these were generally enhanced by weight correction. Differences between groups were present in the $2-4$ month time period only for $C \mathrm{~L}$,st and FRC, but these were no longer statistically significant after weight correction. Differences between groups were present in the 4-8 month time period for FRC and TLC, which remained after weight correction. For the 8-12 month time period, a difference in raw values was present for TLC; after weight correction this difference was no longer statistically significant, but a difference for RV reached statistical significance.

When the flow rates were examined, differences in the 0-2 time period were found in FVC raw values, which was joined after weight correction by differences in PEF and FEF25-75. Significant differences between control and smoke-exposed animals were found in the 8-12 month time period for FEV1 and FEV1/FVC, both with and without weight correction. The effect of smoke on FEV1 and FEV1/FVC seemed to occur between 4 and 8 months ( $p=0.04$ and $p=0.05$, respectively, before Bonferroni correction), whereas the ageing effect in the control animals occurred between 8 and 12 months. A smoke effect on weight was only present between baseline and 2 months.

\section{Predictive value of tests}

To test whether any of the flow tests had predictive value, we examined whether the initial test value, the test value at the 2 month time-point, or the slope of the line between initial and 2 month tests were able to predict the flow test values at the 12 month time-point. No such relationships could be found.

During the course of the experiment, five control and six smoke-exposed rats died, usually because of postanaesthetic bronchospasm. There were no differences with surviving animals either in baseline function or function tests performed prior to death.

\section{Discussion}

This study utilized a longitudinal format, with pulmonary function tests performed at multiple time-points, after first confirming that such tests can be reproducibly performed. We have shown that pulmonary function in the rat normally changes over a 12 month time period, most likely in relation to growth of the animals. This is particularly true of the lung volumes, which increased over time, as did the $C$ L,st. By contrast, although the FEF25-75 and FVC tended to increase slightly over time, the FEV1 and FEV1/FVC decreased during the 8-12 month time period, suggesting an ageing effect. As discussed below, cigarette smoke appears to accelerate this ageing effect, much in the same manner as has recently been found in humans [11].

Although rats have previously been shown to develop emphysema and airflow obstruction after exposure to cigarette smoke [12], no previous study has utilized a longitudinal design with the extensive pulmonary function test analysis reported in the present study. Using this regimen, we have shown that chronic ciga- rette smoke exposure produced airflow obstruction, albeit not to the same degree as that seen in the guinea-pig model [1]. The VC and FVC of the smoke-exposed animals were increased compared to the control animals, findings which coincided with an effect of smoke on increasing the $C \mathrm{~L}$,st. The profile analysis showed that this effect on $C \mathrm{~L}$,st was significant between baseline and 2 months, and between 2 and 4 months. In humans, $\mathrm{VC}$ is usually decreased in patients with chronic obstructive pulmonary disease (COPD); however, the paradoxical increase seen in the animal models [1] can be explained by the fact that the animals are inflated to a certain transpulmonary pressure, rather than by active inspiration. Thus, a small increase in lung $C \mathrm{~L}$,st will result in an increased $\mathrm{VC}$.

Analysis of the flow data showed that, overall, FEV1, decreased between 4 and 12 months, and there was a significant overall decrease in the smoke-exposed animals. The decrease in the smoke-exposed animals appeared to occur earlier compared to the control animals, which showed a decrease between 8 and 12 months. FEV1/FVC also decreased over the total experimental time period, and there was a significant overall effect of smoke exposure, which occurred primarily between 4-8 months. By contrast, in the control animals, the normal decrease in airflow associated with ageing was found between 8 and 12 months.

There is an obvious time disparity between the effect of smoke on the lung volumes and compliance, and the effect of prolonged smoke exposure on airflow, with the former occurring early and the latter occurring later in the exposure time course. Alterations of the lung matrix could be one explanation for this. MACKLEM and EIDELMAN [13] and LAROS and KUYPER [14] have suggested that there is a dynamic alteration of the lung collagen in response to chronic cigarette smoke exposure, which may be manifested in abnormal pulmonary physiology. SNIDER et al. [15] developed a model of emphysema induced by cadmium chloride, and found decreased forced expiratory flow with an increase in lung collagen as measured biochemically. Sugihara et al. [16] examined length-tension curves of lung tissue fragments and demonstrated that emphysematous alveolar walls had increased length, but decreased distensibility. MACKLEM and EIDELMAN [13] showed that patients with COPD had an increased specific elastance and, therefore, required a greater inflation pressure to produce volume increases.

In our guinea-pig model of chronic smoke exposure [1], we have previously shown that the lung parenchymal matrix components are bimodally altered by cigarette smoke exposure, with a decrease in collagen after 1 month of exposure, followed by an increase in collagen matrix components after 6 and 12 months [17]. Physiologically, the animals developed initial alterations in lung volumes, followed in the latter months by decreased flow rates. The present study also shows alterations in lung volumes, with an overall decrease in flow rates. Thus, it seems likely that a similar process of matrix remodelling is occurring in this rat model. Matrix is known to be altered throughout life [18]; the physiological changes seen in the smoke-exposed rats may be analogous to a process of accelerated ageing, such as that found in human cigarette smokers [11]. 
The smoke-exposed animals weighed less than the control animals at all of the time-points. This phenomenon was previously found in our guinea-pig model of chronic cigarette smoke exposure [1], and has recently also been found in rats exposed chronically to nicotine vapour [19]. In humans, there is a known relationship between lung function and somatic growth [20], and we have previously shown that both lung volumes and flow rates are related to weight in the guinea-pig model [3]. When we examined the data using weight as a corrective factor, we found that the majority of the overall effects of time remained significant, and that the profile analysis again suggested that cigarette smoke exerted its effect on lung volumes early in the experimental time course, but between the last two time periods for FEV1 and FEV1/FVC.

Animals, like humans, show a wide degree of susceptibility to deleterious agents [4]. In humans, this produces the "healthy worker" effect, i.e. workers who are particularly susceptible will drop out of the workforce and, hence, not appear in subsequent cross-sectional analyses $[21,22]$. The healthy worker effect, thus, tends to underestimate the adverse effects of specific exposures. Although animals do not have the ability to remove themselves from exposure to test agents, those individuals who are very susceptible may die before the completion of the experiment [2,4], and thus "remove" themselves from any statistical analysis, producing a similar situation. In the present study, a comparison of measured characteristics of the animals who died with those of the living animals at the same time-point, showed no significant differences. Thus, unlike the guinea-pig model, the "healthy smoker" effect is a not a feature of the rat model.

An additional potential feature of a longitudinal analysis is the ability to ascertain whether any one test is able to predict final function [23]. However, one of the problems in using an inbred animal model is that these animals tend to have a stereotypical response to injury. Although there was some variation in the initial pulmonary function tests, all smoke-exposed animals suffered a similar percentage decrement in function by the final test time. Thus, we were unable to find any test of predictive value.

In summary, longitudinal analysis of pulmonary function in the rat model has shown that cigarette smoke exerts its effects early on lung volumes and compliance, while flow alterations occur later, consistent with a picture of accelerated ageing. This type of experimental design should prove useful in better characterizing the time course and nature of cigarette smoking effects.

\section{References}

1. Wright JL, Churg A. Cigarette smoke causes physiologic and morphologic changes of emphysema in the guinea-pig. Am Rev Respir Dis 1990; 142: 1422-1428.

2. Wright JL, Sun J-P. The effect of smoking cessation on pulmonary and cardiovascular function and structure. J Appl Physiol 1994; 76: 2163-2168.

3. Wiggs BR, Churg A, Wright JL. Influence of weight on pulmonary function in the adult guinea-pig. Respiration 1991; 58: 37-41.

4. Wright JL. The relationship of increased pulmonary artery pressure and airflow obstruction to emphysema. $J$ Appl Physiol 1993; 74: 1320-1324.

5. Blouin A, Cormier Y. Endotracheal intubation in guineapigs by direct laryngoscopy. Lab Animal Sci 1987; 37: 244-245.

6. Kujime K, Natelson BH. A method for endotracheal intubation of guinea-pigs. Lab Animal Sci 1981; 31: 715-716.

7. Prignot J. Quantification and chemical markers of tobacco exposure. Eur J Respir Dis 1987; 70: 1-7.

8. Wilkinson L. In: SYSTAT: the system for statistics. Evanston, IL, SYSTAT Inc., 1988.

9. SAS Institute Inc. In: SAS/STAT Users Guide. Cary, NC, SAS Institute, 1985.

10. van Ende CN. Repeated measures analysis: growth and other time-dependent measures. In: Scheiner SM, Gurevitch J, eds. Design and Analysis of Ecological Experiments. New York, Chapman and Hall, 1993.

11. Gold DR, Wang X, Wypij D, Speizer FE, Ware JH, Dockery DW. Effects of cigarette smoking on lung function in adolescent boys and girls. $N$ Engl J Med 1996; 335: 931-937.

12. Huber GL, Davies P, Zwilling GR, et al. A morphologic and physiologic bioassay for quantifying alterations in the lung following experimental chronic inhalation of tobacco smoke. Clin Respir Physiol 1981; 17: 269327.

13. Macklem PT, Eidelman D. Re-examination of the elastic properties of emphysematous lungs. Respiration 1990; 57: 187-192.

14. Laros CD, Kuyper CMA. The pathogenesis of pulmonary emphysema. Respiration 1976; 33: 325-348.

15. Snider GL, Lucey EC, Faris B, Jung-Legg Y, Stone PJ, Franzblau C. Cadmium chloride-induced airspace enlargement with interstitial pulmonary fibrosis is not associated with destruction of lung elastin. Am Rev Respir Dis 1988; 137: 918-923.

16. Sugihara T, Martin CJ, Hildebrandt J. Length-tension properties of alveolar wall in man. J Appl Physiol 1971; 30: 874-878.

17. Wright JL, Churg A. Smoke-induced emphysema in the guinea-pig is associated with morphometric evidence of collagen breakdown and repair. Am J Physiol 1995; 268: L17-L20.

18. Turino GM. The lung parenchyma: a dynamic matrix. Am Rev Respir Dis 1985; 132: 1324-1334.

19. Waldum HL, Nilsen T, Rorvik H, et al. Long-term effects of inhaled nicotine. Life Sci 1996; 58: 13391346.

20. Sherrill DL, Camilli A, Lebowitz MD. On the temporal relationships between lung function and somatic growth. Am Rev Respir Dis 1989; 140: 638-644.

21. Becklake M. Epidemiological evidence of varying susceptibility to inhaled substances. Pharmacogen 1991; 1: 98-101.

22. Becklake MR. Chronic airflow limitation: its relationship to work in dusty occupations. Chest 1985; 88: 608-617.

23. Burrows B, Knudson RJ, Camilli AE, Lyle SK, Lebowitz MD. The "horse-racing effect" and predicting decline in forced expiratory volume in one second from screening spirometry. Am Rev Respir Dis 1987; 135: 788-793. 